\title{
Evaluation of a computer-assisted multi- professional intervention to address \\ lifestyle-related risk factors for overweight and obesity in expecting mothers and their infants: protocol for an effectiveness- implementation hybrid study
}

\author{
Adrienne Alayli ${ }^{1,2^{*}}$ (D) Franziska Krebs ${ }^{1}$, Laura Lorenz ${ }^{1}$, Farah Nawabi ${ }^{1}$, Anne-Madeleine Bau ${ }^{3}$, Isabel Lück ${ }^{3}$,
} Andrea Moreira $^{3}$, Judith Kuchenbecker ${ }^{3}$, Elena Tschiltschke ${ }^{3}$, Michael John ${ }^{4}$, Stefan Klose ${ }^{4}$, Benny Häusler ${ }^{4}$, Christian Giertz ${ }^{4}$, Ulrike Korsten-Reck ${ }^{5}$ and Stephanie Stock ${ }^{1}$

\begin{abstract}
Background: The first 1000 days after conception are a critical period to encourage lifestyle changes to reduce the risk of childhood obesity and early programming of chronic diseases. A healthy lifestyle during pregnancy is also crucial to avoid high post-partum weight retention. Currently, lifestyle changes are not consistently discussed during routine health services in Germany. The objective of this study is to evaluate a novel computer-assisted lifestyle intervention embedded in prenatal visits and infant check-ups. The intervention seeks to reduce lifestylerelated risk factors for overweight and obesity among expecting mothers and their infants.

Methods: The study is designed as a hybrid effectiveness-implementation trial to simultaneously collect data on the effectiveness and implementation of the lifestyle intervention. The trial will take place in eight regions of the German state Baden-Wuerttemberg. Region were matched using propensity score matching. Expecting mothers ( $n=1860$ ) will be recruited before 12 weeks of gestation through gynecological practices and followed for 18 months. During 11 routine prenatal visits and infant check-ups gynecologists, midwives and pediatricians provide lifestyle counseling using Motivational Interviewing techniques. The primary outcome measure is the proportion of expecting mothers with gestational weight gain within the recommended range. To understand the process of implementation (focus group) interviews will be conducted with providers and participants of the lifestyle intervention. Additionally, an analysis of administrative data and documents will be carried out. An economic analysis will provide insights into cost and consequences compared to routine health services.

(Continued on next page)
\end{abstract}

\footnotetext{
* Correspondence: adrienne.alayli@uk-koeln.de

${ }^{1}$ Institute of Health Economics and Clinical Epidemiology, University Hospital of Cologne (IGKE), Cologne, Germany

${ }^{2}$ Federal Centre for Health Education (BZgA), Cologne, Germany

Full list of author information is available at the end of the article
}

(c) The Author(s). 2020 Open Access This article is distributed under the terms of the Creative Commons Attribution 4.0 International License (http://creativecommons.org/licenses/by/4.0/), which permits unrestricted use, distribution, and reproduction in any medium, provided you give appropriate credit to the original author(s) and the source, provide a link to the Creative Commons license, and indicate if changes were made. The Creative Commons Public Domain Dedication waiver (http://creativecommons.org/publicdomain/zero/1.0/) applies to the data made available in this article, unless otherwise stated. 
(Continued from previous page)

Discussion: Findings of this study will add to the evidence on lifestyle interventions to reduce risk for overweight and obesity commenced during pregnancy. Insights gained will contribute to the prevention of early programming of chronic disease. Study results regarding implementation fidelity, adoption, reach and cost-effectiveness of the lifestyle intervention will inform decisions about scale up and public funding.

Trial registration: German Clinical Trials Register (DRKS00013173). Registered 3rd of January 2019, https://www.drks.de

Keywords: Pregnancy, Overweight and obesity prevention, Lifestyle, Gestational weight gain, Multi-professional collaboration, Effectiveness, Implementation, Cost, Diet, Physical activity, Substance use.

\section{Introduction}

Overweight and obesity are increasing worldwide [1]. More than one in two adults and nearly one in six children are overweight or obese in OECD countries [2]. In Germany $35.9 \%$ of the adult population are overweight and $18.1 \%$ are obese [3]. Among children and adolescents $15.4 \%$ are overweight and $5.9 \%$ are obese [4].

The high prevalence of overweight and obesity represents a key risk factor for non-communicable diseases, including cardiovascular diseases, diabetes, musculoskeletal disorders and some cancers [1]. As childhood overweight and obesity tend to persist into adulthood [5], early interventions are essential.

There is growing evidence that lifestyle factors in the first 1000 days after conception are important predictors of childhood overweight and obesity. Maternal gestational weight gain (GWG), smoking and diet have been identified as predictors during pregnancy [6-10]. Rapid infant weight gain, nicotine exposure and infant feeding practices have been identified as essential factors after birth [6, 10-13].

Human epidemiology and animal model studies suggest that exposure to these factors affects developmental processes, which program susceptibility to obesity and other chronic conditions manifesting later in life [14, 15]. Pregnancy and early infancy therefore represent a critical period for targeted prevention efforts.

Lifestyle changes initiated during pregnancy also produce benefits for expecting mothers. Evidence suggests that adequate GWG can avoid high post-partum weight retention and thus reduce the risk of overweight and obesity following pregnancy $[16,17]$.

Several preventive interventions addressing maternal lifestyle during pregnancy have been evaluated. Two meta-analyses show that diet and exercise interventions during pregnancy can effectively reduce excessive gestational weight gain $[18,19]$. There is also evidence that professional-led educational interventions can increase uptake of breastfeeding [20]. A Cochrane review indicates that counseling interventions during pregnancy can effectively increase smoking cessation rates [21]. High postpregnancy relapse rates call for strategies to promote continued abstinence post-partum, however [21, 22].
Lifestyle intervention trials initiated during pregnancy that continue during infancy are scarce [23-25]. They are heterogeneous, have methodological limitations and have produced mixed results [23, 24]. Few intervention studies provide evidence for beneficial effects on growth status of infants or children of obese women only [24].

Interventions targeting multiple lifestyle related risk factors hold promise for more effective childhood obesity prevention $[10,26]$. So far, intervention studies targeting feeding, diet and physical activity behaviors in combination with prenatal nicotine exposure are lacking [23].

The GeMuKi project (acronym for "Gemeinsam Gesund: Vorsorge plus für Mutter und Kind" - Strengthening health promotion: enhanced check-up visits for mother and child) aims to incorporate a brief multifactorial lifestyle intervention into routine prenatal visits and infant check-ups. In Germany, these check-ups currently focus on early identification of diseases and developmental problems only. Existing guidelines for pre- and postnatal care mention that providers have a role in discussing modifiable lifestyle factors, but they do not specify content or format of lifestyle counseling [27].

Recent findings of the GeliS trial (acronym for "Gesund leben in der Schwangerschaft") conducted in the German state of Bavaria suggest that incorporating lifestyle counseling into routine prenatal health services is feasible and leads to high compliance rates [28]. The lifestyle intervention itself achieved only slight improvements in prenatal intake of food items, exclusive breastfeeding behavior and maternal post-partum weight development [29, 30]. By continuing lifestyle counseling after birth and utilizing theoretically underpinned Motivational Interviewing (MI) techniques, the GeMuKi intervention addresses some limitations of the GeliS intervention. In addition, the GeMuKi intervention includes a novel shared telehealth platform to support multi-professional providers in the counseling process with a corresponding App for intervention participants.

The objective of this study is to examine effectiveness of the GeMuKi intervention and explore its potential for widespread implementation. It will answer the following research questions: 
- Does the GeMuKi intervention effectively improve lifestyle-related risk factors for overweight and obesity in expecting mothers and their infants compared to routine practice?

- How does implementation of the GeMuKi intervention take place in practice? What factors facilitate or hinder successful implementation during routine prenatal visits and infant check-ups?

- What costs, health service use and consequences are associated with the GeMuKi intervention from a public payer perspective? How do these compare to routine health practice?

\section{Methods/design}

\section{Study design}

A hybrid effectiveness-implementation trial (Type II) is being used to simultaneously collect data on the effectiveness and implementation of the GeMuKi lifestyle intervention [31, 32]. This design was selected because there is strong evidence that interventions during pregnancy can effectively improve lifestylerelated risk factors, research indicates that lifestyle counseling during routine check-up visits is feasible in Germany and evidence on implementation of lifestyle interventions during pregnancy is scarce. The GeMuKi intervention comprises various components previously identified to enhance lifestyle counseling during pregnancy. To our knowledge, effectiveness of these components has not been evaluated in combination, yet.

The trial has two arms (see Fig. 1). In the intervention arm gynecologists, midwives and pediatricians carry out the GeMuKi lifestyle intervention during routine prenatal visits and infant check-ups. In the control arm they provide care as usual. The study takes place in both urban and rural areas within the German state BadenWuerttemberg. To reduce discrepancies between study regions intervention and control regions were matched into pairs using propensity score matching. Matching was conducted immediately after the project kick off in October 2017 to provide enough time for enrollment of multi-professional providers and for conducting implementation training in the intervention regions before commencing recruitment of study participants. Matching was based on average income per capita, the number of births among persons insured by BARMER (i.e. the statutory health insurer agreeing first to participate in the GeMuKi project) and the number of gynecologists in the study regions. This resulted in four matched study region pairs, which were randomized into intervention and control regions.

Data regarding effectiveness and implementation will be collected at multiple time points over an 18-month study period (see Table 2).

\section{Recruitment procedure}

Recruitment of multi-professional providers commenced in April 2018 and continues until December 2019. For this purpose, informational meetings are being conducted in the study regions. Regional opinion leaders are attending these meetings to raise awareness of the GeMuKi project and promote participation from a user-perspective. Additionally, the project is advertised through professional organizations, journals, conference presentations and through contacting providers directly over the phone and during personal visits.

Recruitment of study participants commenced in January 2019 and continues until September 2020. It takes place during routine prenatal visits conducted in participating gynecologist practices before 12 weeks of gestation. Gynecologists determine eligibility of pregnant women using pre-defined in- and exclusion criteria. They provide eligible women with a project brochure and additional information about the study. For each study participant, who enrolls in the study, gynecologists receive an expense allowance of $20 €$.

\section{In- and exclusion criteria}

Pregnant women are eligible to participate, if they provide informed consent, are $\geq 18$ years old, are $<12$ weeks of gestation at recruitment, are proficient in German language and are enrolled in one of the participating gynecologist practices. To participate in the study, pregnant women also require a health insurance plan from BARMER or from one of the following statutory health insurers, who became project partners upon commencement of the GeMuKi project: AOK Baden-Württemberg, Techniker Krankenkasse and through GWQ Service Plus: Audi BKK, BAHN-BKK, Bertelsmann BKK, BIG direkt gesund, BKK Deutsche Bank AG, BKK Schwarzwald-Baar-Heuberg, BKK Voralb HELLER "Index* LEUZE, Daimler BKK, Die Schwenninger Krankenkasse, energie-BKK, Heimat Krankenkasse, Salus BKK, SBK Siemens-Betriebskrankenkasse, SECURVITA Krankenkasse.

Pregnant women who screen positive for depression (i.e. defined as a sum score of $>9$ or a score $=3$ on item 10 of the Edinburgh Postnatal Depression Scale) are excluded from the study. They are referred to information about the 'Mind: Pregnancy' trial, which takes place simultaneously in the same study regions [33]. It evaluates an intervention to reduce psychological stress during pregnancy. This procedure aims to reduce risk of bias that could be introduced by co-interventions.

Multi-professional computer-assisted lifestyle intervention The development of the GeMuKi intervention has been informed by experiences from the project $9+12$ [34] and the GeliS study [28-30]. It aims to positively influence 


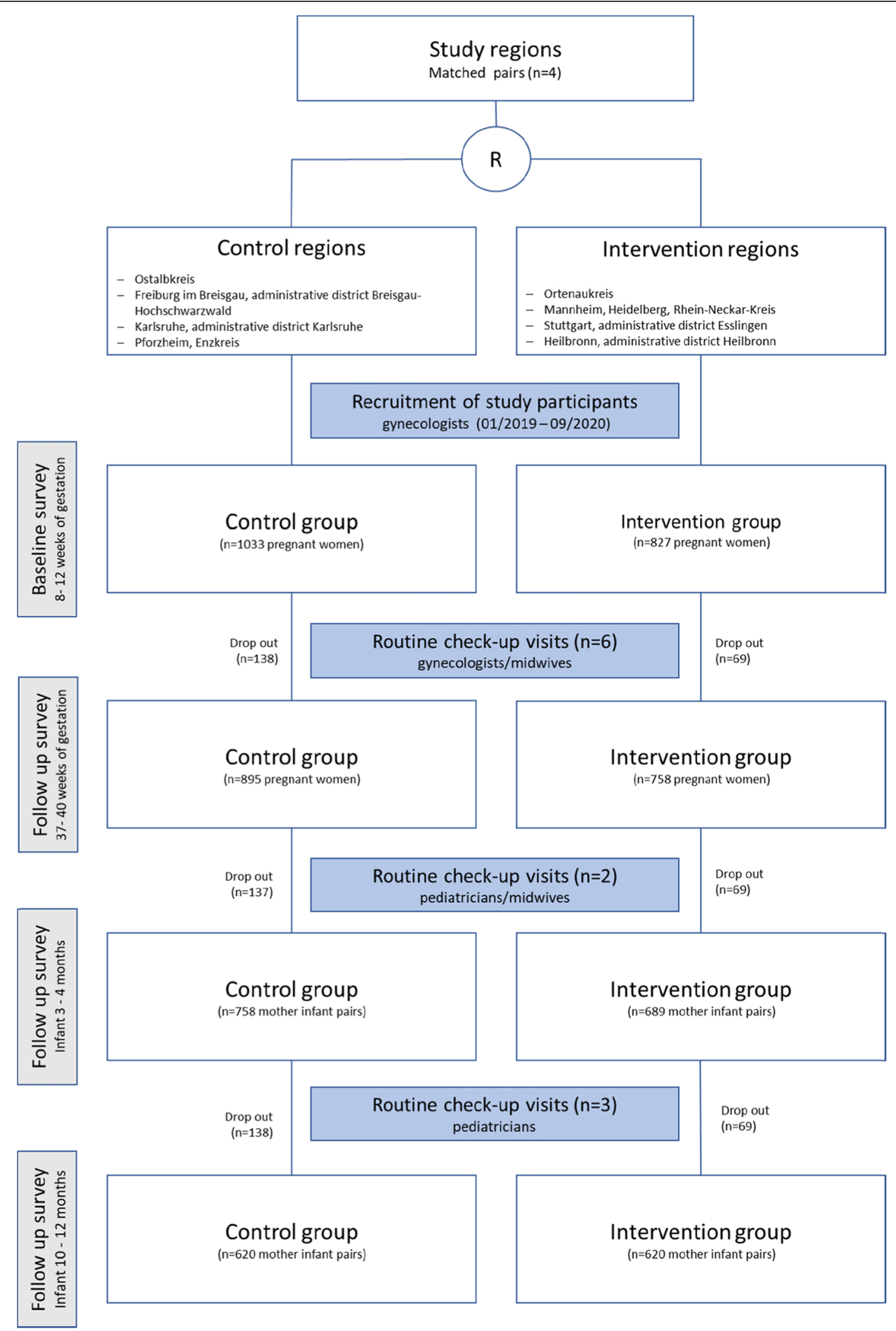

Fig. 1 Study design flow chart 
lifestyle-related risk factors of expecting mothers and their infants. The GeMuKi intervention is designed as a series of brief (approximately $10 \mathrm{~min}$ ) counseling sessions performed by gynecologists, midwives and pediatricians during 11 prenatal and infant check-ups (see Fig. 2). The counseling sessions cover topics relevant during pregnancy and the infant's first year relating to diet, physical activity, breastfeeding, and substance use. Figure 2 provides an overview of the topics addressed over the course of the GeMuKi lifestyle intervention. The topics are based on recently updated national recommendations developed by a multidisciplinary scientific task force $[35,36]$.

Traditionally, behavioral interventions aiming at lifestyle changes rely on providing information and advice. This has proven to be less successful compared to approaches using elements of Motivational Interviewing (MI) to improve communication by health professionals [37, 38].

The GeMuKi intervention takes into consideration that communication of providers should be sensitive to expecting mothers' health literacy in order to have a positive impact on behavior change. Therefore, multiprofessional providers carrying out the GeMuKi intervention receive communication skills training. In addition to the content of the lifestyle intervention itself, the training covers MI techniques. MI is a clientcentered counseling approach designed to enhance motivation for behavioral change by helping clients explore and resolve ambivalence [39].

A key element of MI used in the GeMuKi intervention is agenda mapping. Multi-professional providers employ agenda mapping to focus on a specific topic for lifestyle change (see Fig. 2). For this purpose, they use key message cards with pictograms developed by the Platform Nutrition and Physical Activity (peb) and experienced MI trainers.
After a participant has chosen a topic for lifestyle change, the provider continues the conversation using open-ended questions and then reacts to the participant's answers using reflective listening techniques. Guided by the provider, participants set SMART (Specific Measurable Achievable Reasonable Time Bound) goals for lifestyle change, which can be accomplished until the next check-up visit.

Another objective of the GeMuKi intervention is to increase the level of cooperation between gynecologists, pediatricians and midwives. To achieve this, a novel telehealth platform was developed, which assists providers in the counseling process and enables them to communicate with each other.

\section{Telehealth platform GeMuKi-Assist}

The telehealth platform GeMuKi-Assist has the objective to facilitate cooperation between providers and enhance continuity of lifestyle counseling. It consists of the GeMuKi-Assist Counseling Tool, GeMuKi-Assist App, GeMuKi-Assist Study Monitor and the GeMuKi-Assist Server (see Fig. 3).

Providers and trained practice staff in both intervention and control regions use the GeMuKi-Assist Counseling Tool to enter data routinely documented in the maternity and child medical record booklets. In the intervention regions these data are used to create a GWG curve showing the development of GWG for each individual study participant in relation to the recommended range. The infants' weight progression is displayed by means of percentile curves (see Additional file 1). Providers in the intervention regions also have access to key messages and guiding questions (i.e. standardized content) to support them in carrying out the GeMuKi intervention according to protocol and in alignment with MI

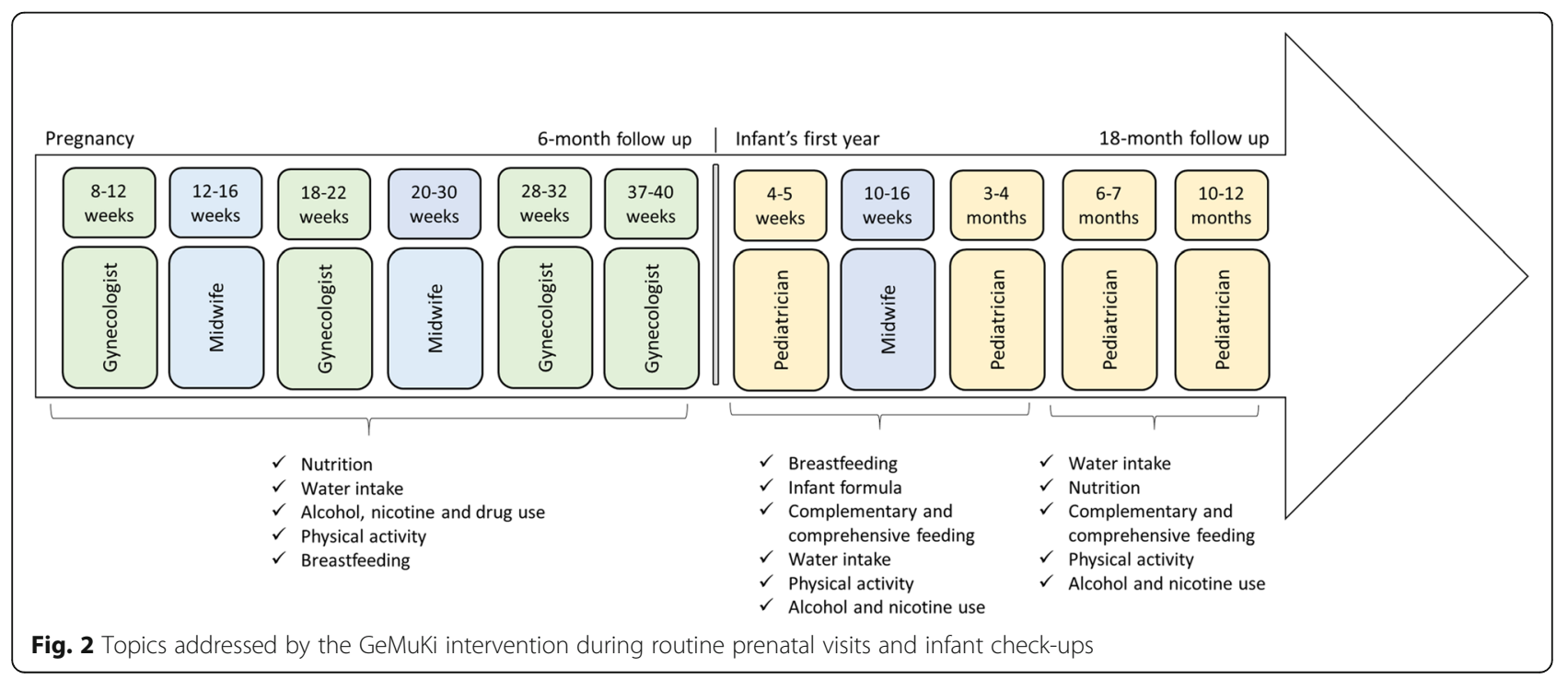




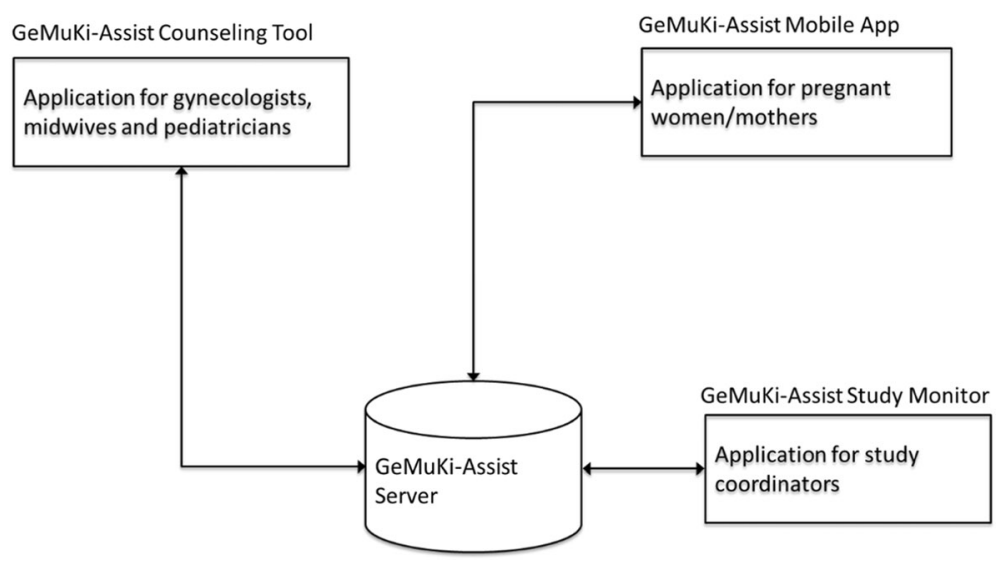

Fig. 3 Overview GeMuKi-Assist Telehealth platform

techniques. They can also document goals for lifestyle change participants want to accomplish until the next check-up visit and have an option to enter notes regarding individual participants. To ensure continuity of the counseling, this information can be accessed by multiprofessional providers involved in the counseling process. Individual goals for lifestyle change entered into the counseling tool are automatically send to the GeMuKi-Assist App as a reminder for study participants.

The GeMuKi-Assist App aims to support intervention group participants in performing lifestyle changes. It provides an overview of individual goals formulated during lifestyle counseling and sends automatic reminders for encouragement (push notifications). The App includes links to reliable sources of information (e.g. institutions providing health education) as well as services and supports available in the region (e.g. psychotherapists and dieticians). An option to conduct automated google keyword searches (e.g. lactation consultant and smoking cessation classes) is also included (see Additional file 1). In addition to these features, which are only available for participants in the intervention group, all participants can use the App for creating personal notes and completion of the electronic surveys in the study.

The GeMuKi-Assist Study Monitor supports the research process alongside the GeMuKi intervention. It is used to create user profiles for providers and study participants and for assigning study participants to corresponding multi-professional providers. Study coordinators also use the tool to monitor the data collection process. Automatic alerts from the GeMuKiAssist server inform them for instance about incomplete data from study participant surveys and data entries in the counseling tool (see Additional file 1).

The GeMuKi-Assist Server handles and saves the data derived from the mobile App, the counseling tool and the study monitor in one central database. Access is controlled for different user groups, who must authorize themselves before accessing the data.

\section{Implementation strategy}

To encourage uptake of the GeMuKi-intervention and implementation as planned an implementation strategy is being used consisting of the three elements: (1) a oneday training for gynecologists, midwives, pediatricians and practice assistants; (2) support by regional study coordinators in participating practices and (3) funding of novel tasks associated with the lifestyle intervention.

The one-day training is conducted before initiating the GeMuKi lifestyle intervention. It covers the basics of MI and the previously mentioned updated national recommendations for a health-promoting lifestyle during pregnancy and the infant's first year. The training material includes a PowerPoint presentation, key message cards as well as brochures and stickers for the maternity and child medical record booklets. The presentation provides information on the purpose of the lifestyle intervention and key messages for a health-promoting lifestyle. It also summarizes the most relevant aspects of the evaluation study conducted alongside the intervention. In addition, the fundamentals of MI are introduced and the implementation of the GeMuKi intervention using selected MI elements explained. Knowledge of theoretical concepts are applied practically through role-play exercises and reinforced by videos with MI examples. The training also covers how to use the GeMuKi-Assist Counseling Tool. The training is carried out by experienced MI trainers from the Healthy Start-Young Family Network (Gesund ins Leben-Netzwerk Junge Familie). The training materials were developed based on the content of the curriculum of the Healthy Start-Young Family Network [40] and additional literature [41-43].

Regional study coordinators provide ongoing support to participating providers over the phone and during 
regular practice visits. They conduct a hands-on introduction to GeMuKi-Assist in the participating providers' practices in both intervention and control regions and answer questions to help solve technical issues with GeMuKi-Assist and other local implementation challenges. They also provide information and advice to encourage protocol compliance (e.g. regarding weighing during pregnancy and flawless documentation). Furthermore, they perform data management. In case of missing data or data error, they contact the respective providers.

All providers participating in the study receive funding for implementing the GeMuKi intervention. They sign a contract with the participating health insurers and the Association of Statutory Health Insurance Physicians of Baden-Württemberg (KVBW). This contract forms the legal basis for the billing process. Providers in the intervention regions can bill $15 €$ per lifestyle counseling session. Providers in both the intervention and control regions can bill $5 €$ per documentation in GeMuKi Assist. Gynecologists and pediatricians in the intervention regions can receive up to $80 €$ and midwives up to $60 €$ per study participant when they carry out all counseling sessions in the study period (see Fig. 2).

\section{Data sources}

Data will be collected at various points in time using multiple methods. Data sources include an electronic survey completed by study participants in the GeMuKiAssist App at four points in time, data entered into the GeMuKi-Assist counseling tool during routine prenatal visits and infant check-ups, (focus group) interviews with multi-professional providers and intervention participants, statutory health insurance claims data and documents. At baseline, study participants also complete a short paper survey including demographic questions.

The selection of data sources was guided by the REAIM framework, which has been developed for evaluation of effectiveness and implementation of interventions in real-world settings [44, 45]. Table 1 provides a summary of constructs that will be measured for each dimension of the RE-AIM framework and data sources used.

\section{Measures to assess effectiveness of the lifestyle intervention}

Outcomes used to assess are described below. Table 2 provides a summary of the points of measurement and data collection methods.

\section{Maternal weight}

During every prenatal visit maternal weight is routinely measured and documented in the maternity record booklet (see Table 2). GWG is calculated as the difference between self-reported pre-pregnancy weight documented during the first prenatal visit and weight at the last prenatal visit.

Excessive GWG is defined according to recommendations of the Health and Medicine Division of the National Academies of Science, Engineering and Medicine (previously known as Institute of Medicine, IOM). These recommendations differ depending on pre-pregnancy Body Mass Index (BMI). For underweight women $(\mathrm{BMI}<18.5)$ the recommended weight gain ranges from 12.5 to $18 \mathrm{~kg}$, for normal weight women (BMI = 18.5-24.9) from 11.5 and $16 \mathrm{~kg}$, for overweight women (BMI = 25-29.9) from 7 to $11.5 \mathrm{~kg}$ and for obese women $(B M I \geq 30)$ from 5 to $9 \mathrm{~kg}$ [46]. Weight gain above the recommended range is classified as excessive GWG. This definition of excessive GWG is similar to the definition used in German guidelines, which currently recommend a maximum weight gain of $16 \mathrm{~kg}$ for normal weight women and a maximum of $10 \mathrm{~kg}$ for overweight and obese women [36]. To assess postnatal weightretention, maternal weight data will also be collected 1 year after birth.

\section{Maternal lifestyle behaviors}

Physical activity behavior during pregnancy will be measured using the Pregnancy Physical Activity Questionnaire (PPAQ) [47]. Maternal smoking behavior and alcohol consumption will be measured using questions from the German Health Interview and Examination Survey for Children and Adolescents (KIGGS) [48]. Dietary behavior will be assessed with a modified version of the Food Frequency Questionnaire used in the German Health Examination Survey for Adults (DEGS), which measures frequency and portion size of the main food groups consumed over the past 4 weeks [49].

\section{Maternal knowledge}

To assess the ability of the lifestyle intervention to increase maternal knowledge of health promoting lifestyle aspects addressed during brief counseling, the research team developed specific knowledge questions. These questions are based on key messages included in the previously mentioned national recommendations for a health-promoting lifestyle during pregnancy and the infant's first year [35, 36]. Data on study participants' health literacy will be collected as part of a separate study component, which will be reported elsewhere.

\section{Infant weight development and body composition}

Infant weight and length will be routinely assessed during infant check-ups. Infant BMI will be calculated and compared with age-specific reference values. The German Kromeyer-Hauschild reference system [50] will be used, because national reference data are more suitable for diagnosis of childhood overweight and obesity [4, 
Table 1 Data sources and measured constructs aligned with RE-AIM dimensions

\begin{tabular}{|c|c|c|c|}
\hline Dimension & Definition & Measured construct & Data source \\
\hline Reach & $\begin{array}{l}\text { The absolute number, proportion, and } \\
\text { representativeness of individuals who } \\
\text { are willing to participate in an initiative, } \\
\text { intervention, or program. }\end{array}$ & $\begin{array}{l}\text { Number and characteristics of participants } \\
\text { and non-participants, reasons for non- } \\
\text { participation }\end{array}$ & $\begin{array}{l}\text { Administrative data in GeMuKi-Assist, focus } \\
\text { groups with multi-professional providers, } \\
\text { paper survey }\end{array}$ \\
\hline \multirow[t]{2}{*}{ Effectiveness } & \multirow{2}{*}{$\begin{array}{l}\text { The impact of an intervention on } \\
\text { important outcomes, including potential } \\
\text { negative effects, quality of life, and } \\
\text { economic outcomes. }\end{array}$} & $\begin{array}{l}\text { Proportion of participants with excessive } \\
\text { weight gain, infant body composition and } \\
\text { weight development }\end{array}$ & Administrative data in GeMuKi-Assist \\
\hline & & $\begin{array}{l}\text { Maternal lifestyle, knowledge, infant } \\
\text { feeding, infant diet and physical activity }\end{array}$ & Electronic survey \\
\hline Adoption & $\begin{array}{l}\text { The absolute number, proportion, and } \\
\text { representativeness of settings and } \\
\text { intervention agents (people who deliver } \\
\text { the program) who are willing to initiate } \\
\text { a program. }\end{array}$ & $\begin{array}{l}\text { Proportion and characteristics of } \\
\text { participating multi-professional practices, } \\
\text { reasons for non-participation and drop-out } \\
\text { of practices }\end{array}$ & $\begin{array}{l}\text { Administrative data in GeMuKi-Assist, docu- } \\
\text { ments and publicly available statistics }\end{array}$ \\
\hline \multirow[t]{4}{*}{ Implementation } & \multirow{3}{*}{$\begin{array}{l}\text { Setting level: the intervention agents' } \\
\text { fidelity to the various elements of an } \\
\text { intervention's protocol, including } \\
\text { consistency of delivery as intended and } \\
\text { the time and cost of the intervention. }\end{array}$} & $\begin{array}{l}\text { Implementation of brief lifestyle advice } \\
\text { intervention (how and by whom?) }\end{array}$ & $\begin{array}{l}\text { Focus groups with multi-professional } \\
\text { providers, }\end{array}$ \\
\hline & & $\begin{array}{l}\text { Intervention costs: human resources and } \\
\text { time, health service use, implementation } \\
\text { costs and training }\end{array}$ & $\begin{array}{l}\text { Administrative data in GeMuKi-Assist, inter- } \\
\text { views with study participants, social health } \\
\text { insurance claims data, documents }\end{array}$ \\
\hline & & $\begin{array}{l}\text { Utilization of the GeMuKi Assist Counseling } \\
\text { Tool, local adaptations of the intervention }\end{array}$ & $\begin{array}{l}\text { Focus groups with multi-professional pro- } \\
\text { viders, interviews with study participants, } \\
\text { administrative data in GeMuKi-Assist }\end{array}$ \\
\hline & $\begin{array}{l}\text { Individual level: the clients' use of the } \\
\text { intervention strategies. }\end{array}$ & $\begin{array}{l}\text { Utilization of GeMuKi-Assist App, goal set- } \\
\text { ting, links etc. } \\
\text { Attainment of lifestyle change goals }\end{array}$ & $\begin{array}{l}\text { Interviews with study participants, } \\
\text { administrative data in GeMuKi-Assist }\end{array}$ \\
\hline \multirow[t]{2}{*}{ Maintenance } & $\begin{array}{l}\text { Setting level: the extent to which a } \\
\text { program or policy becomes } \\
\text { institutionalized or part of the routine } \\
\text { organizational practices and policies. }\end{array}$ & $\begin{array}{l}\text { Providers becoming experienced in } \\
\text { delivering lifestyle advice, lifestyle advice } \\
\text { becoming a routine component of practice } \\
\text { processes }\end{array}$ & $\begin{array}{l}\text { Focus groups with multi-professional pro- } \\
\text { viders, administrative data in GeMuKi-Assist }\end{array}$ \\
\hline & $\begin{array}{l}\text { Individual level: the long-term effects of } \\
\text { a program on outcomes after } 6+ \\
\text { months after the most recent interven- } \\
\text { tion contact. }\end{array}$ & $\begin{array}{l}\text { Maintenance of lifestyle changes and } \\
\text { weight, drop out of study participants }\end{array}$ & $\begin{array}{l}\text { Administrative data in GeMuKi-Assist, } \\
\text { electronic survey }\end{array}$ \\
\hline
\end{tabular}

51]. To allow for comparisons with international research, the research team will also compare infant weight and length measures with WHO Growth Standards [52].

\section{Infant feeding, diet and physical activity}

Breastfeeding will be routinely documented in the GeMuKi-Assist counseling tool during infant check-ups. At the age of 10 to 12 months study participants will complete a modified version of the food frequency questionnaire used in the German Health Interview and Examination Survey for Children and Adolescents (KIGGS) [53]. It measures frequency and portion sizes of main food groups infants consumed over the past 4 weeks. Additionally, parental feeding practices will be examined with single items from the Comprehensive Feeding Practices Questionnaire (CFPQ) [54]. Study participants will also complete several questions on their infants' physical activity behavior developed by the research team.

\section{Evaluation of the implementation process}

To gain insights into the implementation process, the study team will examine which components of the lifestyle intervention are implemented as planned and which components are being modified. For this purpose, focus groups and interviews with multi-professional providers and study participants will be carried out. Additionally, data entered into the GeMuKi-Assist Counseling Tool will be analyzed. Among other variables, the research team will analyze counseling contents, characteristics of participating providers, characteristics of expecting women and infants reached by the intervention and the total number of lifestyle counseling sessions provided. Finally, documents will be analyzed, such as minutes taken during implementation training.

Qualitative interviews and focus groups will provide insights into factors facilitating and hindering implementation from the perspective of providers and participants in the lifestyle counseling. These qualitative data will also shed light on contextual factors influencing the 
Table 2 Outcome measures at baseline and follow up

\begin{tabular}{|c|c|c|c|c|c|c|c|c|c|c|}
\hline & \multicolumn{4}{|c|}{ Pregnancy } & \multicolumn{6}{|c|}{ Infant's first year } \\
\hline & $\begin{array}{l}8-12 \\
\text { weeks }\end{array}$ & $\begin{array}{l}18-22 \\
\text { weeks }\end{array}$ & $\begin{array}{l}28-32 \\
\text { weeks }\end{array}$ & $\begin{array}{l}37-40 \\
\text { weeks }\end{array}$ & At birth & $\begin{array}{l}3-10 \\
\text { days }\end{array}$ & $\begin{array}{l}4-5 \\
\text { weeks }\end{array}$ & $\begin{array}{l}3-4 \\
\text { months }\end{array}$ & $\begin{array}{l}6-7 \\
\text { months }\end{array}$ & $\begin{array}{l}10-12 \\
\text { months }\end{array}$ \\
\hline Maternal weight ${ }^{a}$ & $x$ & $x$ & $x$ & $x$ & & & & & & $x$ \\
\hline Maternal physical activity & $x$ & & & $x$ & & & & $x$ & & \\
\hline Maternal smoking & $x$ & & & $x$ & & & & $x$ & & $x$ \\
\hline Maternal alcohol use & $x$ & & & $x$ & & & & $x$ & & $x$ \\
\hline Maternal diet & $x$ & & & $x$ & & & & $x$ & & \\
\hline Maternal knowledge & $x$ & & & $x$ & & & & $x$ & & $x$ \\
\hline Breastfeeding ${ }^{a}$ & & & & & & & $x$ & $x$ & $x$ & $x$ \\
\hline Infant weight and length ${ }^{a}$ & & & & & $x$ & $x$ & $x$ & $x$ & $x$ & $x$ \\
\hline Infant nutrition & & & & & & & & & & $x$ \\
\hline Infant physical activity & & & & & & & & $x$ & & $x$ \\
\hline
\end{tabular}

Notes: $\mathrm{a}=$ data are routinely collected and transferred into GeMuKi-Assist during check-up visits, all other measures are collected by an electronic self-report survey. Please note that this table only includes check-up visits, in which providers assess the specified outcomes

implementation process and outcomes for expecting mothers or their infants. To examine dynamic changes over time the research team will conduct interviews and focus groups both at the beginning and the end of the implementation process.

The evaluation of the implementation process will be informed by the Tailored Implementation for Chronic Diseases (TICD) checklist. This checklist is based on a synthesis of frameworks and taxonomies of determinants of professional practice [55]. It identifies determinants that influence professional practice in seven domains: guideline factors, individual health professional factors, patient factors, professional interactions, incentives and resources, capacity for organizational change, social political and legal factors. The checklist will guide the choice of measures used to understand factors influencing adoption, implementation and maintenance of the GeMuKi intervention by multi-professional providers.

\section{Economic evaluation}

A cost-consequence analysis will be performed, because the GeMuKi intervention seeks to modify multiple outcomes in expecting mothers, their infants and at the system level. Cost-consequence analyses compare costs and consequences of alternatives in a disaggregated manner [56]. This provides greater transparency to decision makers, who want to weigh multiple aspects against each other $[57,58]$.

The analysis will be conducted from a health insurance perspective. Cost components considered in the analysis include intervention costs, health service use and implementation costs. Intervention and implementation costs will be calculated based on documentation of personnel time and other resources used. Service use will be calculated using social health insurance claims data. These data include in- and outpatient treatment, medication use, aids and remedies, use of preventive services and sick leave periods. Outcomes considered in the analysis will include the above described lifestyle-related risk factors for overweight and obesity in expecting mothers and their infants. Additionally, outcomes at the systemlevel will be considered, such as changes in collaboration practices between multi-professional providers. These will be derived from qualitative data analyses conducted to gain understanding of implementation processes.

\section{Sample size calculation}

GWG was used as primary outcome for the sample size calculation, because healthy GWG is discussed with all expectant mothers participating in the lifestyle intervention. The brief lifestyle intervention is assumed to reduce the proportion of study participants with excessive gestational weight gain by $10 \%$. Similar interventions have achieved a reduction in the proportion of excessive weight gain of around $20 \%[18,59]$. The target was set lower in this study, because the lifestyle intervention is implemented in a routine health service setting with less stringent inclusion criteria. To detect a $10 \%$ reduction in excessive gestational weight gain with a power of $80 \%$, an alpha of 0.05 and an ICC of 0.05 a sample of $n=1240$ pregnant women is required. This number was increased to $n=1860$ to account for a drop-out rate of $25 \%$ in the intervention group and a $40 \%$ drop-out rate in the control group (see Fig. 1).

\section{Data analyses}

The data entry fields in the GeMuKi-Assist Counseling Tool and electronic surveys collected through the GeMuKi-Assist App are predefined to allow for plausible data only. Additional plausibility checks will be performed before commencing data analysis. Analyses of these quantitative data using descriptive statistics, 
statistical tests and regression models will be conducted in SPSS and R. Analyses for all primary and secondary outcomes will follow an intention-to-treat principle, which compares the intervention arm to the control arm, without regard to intervention completion or compliance. Mixed effects models will be used to account for the clustered structure of the data. Multiple imputation methods will be used to deal with missing values. Exploratory analyses will be performed to explore intervention outcomes for subgroups of study participants, e.g. according to SES and migration background.

All focus groups and interviews will be audio-recorded and transcribed verbatim. Qualitative analysis of focus groups, interviews and documents will be carried out in MAXQDA using a framework analysis approach [60]. Two multidisciplinary researchers will conduct coding independently and discuss discrepancies. The principle of triangulation will be applied continuously to test validity through comparing information from different data sources.

To provide a better understanding of the overall process of implementation and gain insights into possible interactions between implementation and effectiveness of the GeMuKi intervention the research team will conduct integrated data analyses combining qualitative and quantitative data sources.

\section{Discussion}

This study will evaluate a brief counseling intervention to reduce lifestyle-related risk factors of overweight and obesity among expectant mothers and their infants. The GeMuKi intervention is innovative, as it combines several components that have been identified to enhance lifestyle counseling during pregnancy.

First, the lifestyle counseling is integrated into routine prenatal visits and infant check-ups. This puts a smaller burden on participants than add-on approaches [61] and provides a low threshold approach to reach expecting mothers and their infants. According to most recent estimates almost $90 \%$ of expecting mothers in Germany regularly attend prenatal visits [62] and over $95 \%$ of infants attend infant check-ups during the first year of life [63].

Second, lifestyle counseling is tailored to individual intervention participants. A tailored approach that recognizes individual differences in motivation, knowledge, needs and circumstances is recommended, because onesize fits all approaches have shown to be less effective in preventing overweight and obesity $[61,64]$. The GeMuKi intervention consists of a series of brief counseling sessions using MI techniques. MI is a person-centered counseling approach, which encourages active involvement of intervention participants in the behavior change process. As evidenced by systematic reviews, MI has effectively promoted different health behaviors [65, 66] and has been associated with lifestyle changes in the long-term [67].

Third, providers implementing the GeMuKi intervention, will receive training in applying MI techniques. This will address needs expressed by professionals providing pre- and postnatal care to improve communication skills to discuss the sensitive topic of obesity and gestational weight gain [68-71].

Fourth, lifestyle counseling in the GeMuKi intervention will be supported by the novel telehealth platform GeMuKi-Assist. It includes a counseling tool for documentation and collaboration between multi-professional providers, an App for study participants with supporting information to encourage attainment of lifestyle change goals and a study monitor to support the evaluation study. An increasing body of evidence suggests that when used as an adjunct to face-to-face counseling methods computer and communication technology can be an effective tool to achieve lifestyle behavior changes, also among women with lower socio-economic status [72, 73].

Finally, the GeMuKi lifestyle counseling will be provided continuously over an 18-month period. This is in line with previous research findings, demonstrating that longer duration of lifestyle interventions result in more effects $[74,75]$.

The GeMuKi intervention will be evaluated in eight regions of the German state Baden-Wuerttemberg. To support implementation as planned, a comprehensive implementation strategy has been developed. It includes a training curriculum and funding scheme, which can be scaled up, in case the intervention proves to be effective. This evaluation study is designed to provide insights for policy makers at the German Federal Joint Committee (G-BA), who will decide about roll-out and public funding of the intervention on a federal scale.

\section{Strengths of the study}

The effectiveness-implementation hybrid design will concurrently provide insights into effectiveness of the GeMuKi intervention and the process of implementation. It combines design features from a pragmatic clinical trial with concepts from implementation research in order to facilitate a more rapid translation of research evidence into practice [31]. Guided by the RE-AIM framework, various data sources will be used to add further context to findings on effectiveness of the GeMuKi intervention. The study will provide information about factors that influence adoption of the intervention by multi-professional providers, reach of the target group, implementation fidelity and costs. Both from the perspective of providers as well as intervention participants the study will identify ways to optimize the intervention 
to enhance effectiveness, client satisfaction and ease of implementation.

The 18-month follow up is a second strength of this study. Expecting mothers will be included in the study before 12 weeks of gestation and will participate in the GeMuKi intervention until 1 year after birth. The study findings will add to the limited evidence from intervention studies aimed at reducing risk of childhood overweight and obesity, which are commenced during pregnancy and continued after birth [23-25]. They will increase our understanding of effective early intervention strategies to prevent early programming of chronic disease.

\section{Challenges and limitations}

Execution of this study protocol involves several challenges. Embedding the GeMuKi intervention into routine care may pose a challenge for providers, who already have limited time during busy patient schedules [61]. To support providers in conducting the lifestyle counseling efficiently and as planned, the GeMuKi-Assist Counseling Tool includes various supports for providers, such as example questions to discuss with women.

Detecting expected effects of the GeMuKi intervention will require a large sample size. To address this challenge, multiple recruitment strategies will be used. To encourage intervention uptake by multi-professional providers the research team will involve regional opinion leaders among professional groups. Additionally, a relatively high drop-out rate was assumed in the power calculation.

An intervention provided in health service settings can only have a limited impact on individual lifestyle behaviors. Important other determinants in the social, physical and economic environment are not directly addressed by the GeMuKi intervention. Study participants can only be referred to additional supports and resources available in the community. Hence, the GeMuKi intervention can only be one element in an integrated, system wide approach required for successful obesity prevention.

\section{Supplementary information}

Supplementary information accompanies this paper at https://doi.org/10. 1186/s12889-020-8200-4.

Additional file 1. GeMuKi-Assist Telehealth Platform - additional

information and illustrations.

\section{Abbreviations}

BMI: Body Mass Index; GeMuKi: Gemeinsam Gesund Vorsorge Plus für Mutter und Kind (Strengthening health promotion: enhanced check-up visits for mother and child); GWG: Gestational weight gain; MI: Motivational Interviewing; OECD: Organisation for Economic Co-operation and Development

\section{Acknowledgements}

This work was supported by the Innovation Fund of the Federal Joint Committee, the G-BA (Project no. 01NVF17014) and is carried out by a consortium of five partners: Plattform Ernährung und Bewegung, Institut für Gesundheitsökonomie und Klinische Epidemiologie Universitätsklinikum Köln, Fraunhofer Institut für Offene Kommunikationssysteme FOKUS, BARMER, Kassenärztliche Vereinigung Baden-Wuerttemberg. The authors would like to thank Cornelia Wäscher and Kristina Lorrek for their contributions to the grant proposal. We gratefully acknowledge Thomas Kauth for contributing to the initial project idea and for his support during the implementation. We would also like to thank Brigitte Neumann and Sonja Eichin for developing and conducting the training in all intervention regions. Andrea Seifarth, Denise Torricella, Svenja Oort und Hilke Friesenborg coordinated the study in the study regions in Baden-Wuerttemberg. We acknowledge the members of the scientific advisory committee: Hans Hauner, Joachim Dudenhausen, Liane Schenk, Julika Loss and Andrea Lambeck. Finally, we would like to thank the following partners, who have an essential role for the success of the GeMuKi project: Gesund ins Leben - Netzwerk Junge Familie, Berufsverband der Kinder- und Jugendärzte, Berufsverband der Frauenärzte, Hebammenverband Baden-Wuerttemberg, Kassenärztliche Vereinigung Baden-Wuerttemberg, Landesärztekammer Baden-Wuerttemberg, Universität Freiburg, Barmer, AOK Baden-Wuerttemberg, Techniker Krankenkasse and all other health insurers participating in the project through GWQ Service Plus.

\section{Author's contributions}

The GeMuKi project is carried out by a consortium, which is coordinated by AMB. SS, AA, FK, LL, FN are members of the research team and contributed to the design of the study. AA and SS developed the study protocol. AMB, IL, AM, JK, ET developed the enhanced lifestyle intervention. IL, JK, AM and ET coordinated the study in the study regions in Baden-Wuerttemberg. MJ, SK, BH and CG were responsible for the development of the GeMuKi-Assist Telehealth Platform. AA wrote the manuscript. All authors provided comments and approved the final manuscript.

\section{Funding}

This study is funded by the Innovation Fund of the Federal Joint Committee (G-BA), Module 3: Improving communication with patients and promoting health literacy (Project no. 01NVF17014). The study protocol has passed a review process.

\section{Availability of data and materials Not applicable.}

\section{Ethics approval and consent to participate}

Ethical approval to conduct this study was obtained from the University Hospital of Cologne Research Ethics committee (ID:18-163) and the State Chamber of Physicians in Baden-Wuerttemberg (ID: B-F-2018-100). Study data will only be processed in a pseudonymized form in accordance with the EU General Data Protection Regulation (GDPR). Written informed consent will be obtained from all study participants at baseline.

\section{Consent for publication}

Not applicable.

\section{Competing interests}

The authors declare that they have no competing interests.

\section{Author details}

${ }^{1}$ Institute of Health Economics and Clinical Epidemiology, University Hospital of Cologne (IGKE), Cologne, Germany. ${ }^{2}$ Federal Centre for Health Education (BZgA), Cologne, Germany. ${ }^{3}$ Platform Nutrition and Physical Activity (peb), Berlin, Germany. ${ }^{4}$ Fraunhofer Institute for Open Communication Systems (FOKUS), Berlin, Germany. ${ }^{5}$ Adiposity-Academy Freiburg, Freiburg, Germany.

Received: 7 January 2020 Accepted: 10 January 2020

Published online: 15 April 2020

\section{References}

1. WHO. Obesity and overweight. 2018 [30.10.2019]. Available from: https:// www.who.int/en/news-room/fact-sheets/detail/obesity-and-overweight. 
2. OECD. Obesity Update 2017. Available from: https://www.oecd.org/els/ health-systems/Obesity-Update-2017.pdf.

3. Schienkiewitz A, Mensink $G$, Kuhnert $R$, Lange $C$. Übergewicht und Adipositas bei Erwachsenen in Deutschland. J Health Monit. 2017;2:21-8.

4. Schienkiewitz A, Damerow S, Schaffrath RA. Prevalence of underweight, overweight and obesity among children and adolescents in Germany. KiGGS wave 2 results according to international reference systems. J Health Monit. 2018;3(3):56-69.

5. Singh AS, Mulder C, Twisk JWR, Van Mechelen W, Chinapaw MJM. Tracking of childhood overweight into adulthood: a systematic review of the literature. Obes Rev. 2008;9(5):474-88.

6. Iguacel I, Chung A, Gearon E, Moreno LA, Peeters A, Backholer K. Influence of early-life risk factors on socioeconomic inequalities in weight gain. J Public Health. 2018:40(4):447-55.

7. Ensenauer R, Chmitorz A, Riedel C, Fenske N, Hauner H, Nennstiel-Ratzel U, et al. Effects of suboptimal or excessive gestational weight gain on childhood overweight and abdominal adiposity: results from a retrospective cohort study. Int J Obes. 2013;37(4):505-12.

8. Rayfield S, Plugge E. Systematic review and meta-analysis of the association between maternal smoking in pregnancy and childhood overweight and obesity. J Epidemiol Community Health. 2017;71(2):162-73.

9. Mourtakos SP, Tambalis KD, Panagiotakos DB, Antonogeorgos G, Arnaoutis $G$, Karteroliotis K, et al. Maternal lifestyle characteristics during pregnancy, and the risk of obesity in the offspring: a study of 5,125 children. BMC Pregnancy Childbirth. 2015;15(1):66.

10. Woo Baidal JA, Locks LM, Cheng ER, Blake-Lamb TL, Perkins ME, Taveras EM. Risk factors for childhood obesity in the first 1,000 days: a systematic review. Am J Prev Med. 2016;50(6):761-79.

11. Owen CG, Martin RM, Whincup PH, Smith GD, Cook DG. Effect of infant feeding on the risk of obesity across the life course: a quantitative review of published evidence. Pediatrics. 2005;115(5):1367-77.

12. Lisboa P, Oliveira E, Moura E. Obesity and endocrine dysfunction programmed by maternal smoking in pregnancy and lactation. Front Physiol. 2012;3(437):1-9.

13. Yang Z, Huffman SL. Nutrition in pregnancy and early childhood and associations with obesity in developing countries. Matern Child Nutr. 2012; 9(S1):105-19.

14. Taylor PD, Poston L. Developmental programming of obesity in mammals. Exp Physiol. 2007;92(2):287-98.

15. Pico C, Palou A. Perinatal programming of obesity: an introduction to the topic. Front Physiol. 2013;4:255-8.

16. Rong K, Yu K, Han X, Szeto IM, Qin X, Wang J, et al. Pre-pregnancy BMI, gestational weight gain and postpartum weight retention: a meta-analysis of observational studies. Public Health Nutr. 2015;18(12):2172-82.

17. Nehring I, Schmoll S, Beyerlein A, Hauner H, von Kries R. Gestational weight gain and long-term postpartum weight retention: a meta-analysis. Am J Clin Nutr. 2011;94(5):1225-31.

18. Muktabhant B, Lawrie TA, Lumbiganon P, Laopaiboon M. Diet or exercise, or both, for preventing excessive weight gain in pregnancy. Cochrane Database Syst Rev. 2015;(6):1-207. https://doi.org/10.1002/14651858. CD007145.pub3.

19. Craemer KA, Sampene E, Safdar N, Antony KM, Wautlet CK. Nutrition and exercise strategies to prevent excessive pregnancy weight gain: a metaanalysis. AJP Rep. 2019;09(01):92-120.

20. Balogun OO, O'Sullivan EJ, McFadden A, Ota E, Gavine A, Garner CD, et al. Interventions for promoting the initiation of breastfeeding. Cochrane Database Syst Rev. 2016;11:1-100.

21. Chamberlain C, O'Mara-Eves A, Porter J, Coleman T, Perlen SM, Thomas J, et al. Psychosocial interventions for supporting women to stop smoking in pregnancy. Cochrane Database Syst Rev. 2017;10(2):1-425.

22. Coleman-Cowger VH. Smoking cessation intervention for pregnant women: a call for extension to the postpartum period. Matern Child Health J. 2012;16(5):937-40,

23. Blake-Lamb TL, Locks LM, Perkins ME, Woo Baidal JA, Cheng ER, Taveras EM. Interventions for childhood obesity in the first 1,000 days. A systematic review. Am J Prev Med. 2016;50(6):780-9.

24. Dalrymple KV, Martyni-Orenowicz J, Flynn AC, Poston L, O'Keeffe M. Can antenatal diet and lifestyle interventions influence childhood obesity? A systematic review. Matern Child Nutr. 2018;14(4):1-17.

25. Redsell SA, Edmonds B, Swift JA, Siriwardena AN, Weng S, Nathan D, et al. Systematic review of randomised controlled trials of interventions that aim to reduce the risk, either directly or indirectly, of overweight and obesity in infancy and early childhood. Matern Child Nutr. 2016;12(1):24-38.

26. Gilman SE, Loucks EB. Invited commentary: does the childhood environment influence the association between every $\mathrm{x}$ and every $\mathrm{y}$ in adulthood? Am J Epidemiol. 2012;176(8):684-8.

27. Gemeinsamer Bundesausschuss (G-BA). Richtlinien des Gemeinsamen Bundesausschusses über die ärtliche Betreuung während der Schwangerschaft und nach der Entbindung ("Mutterschafts-Richtlinien"); 2019. p. 1-38.

28. Kunath J, Günther J, Rauh K, Hoffmann J, Stecher L, Rosenfeld E, et al. Effects of a lifestyle intervention during pregnancy to prevent excessive gestational weight gain in routine care - the cluster-randomised Gelis trial. BMC Med. 2019;17(1):5.

29. Günther J, Hoffmann J, Kunath J, Spies M, Meyer D, Stecher L, et al. Effects of a lifestyle intervention in routine care on prenatal dietary behavior-findings from the cluster-randomized Gelis trial. J Clin Med. 2019:8(7):1-15.

30. Hoffmann J, Günther J, Stecher L, Spies M, Meyer D, Kunath J, et al. Effects of a lifestyle intervention in routine care on short- and long-term maternal weight retention and breastfeeding behavior-12 months follow-up of the cluster-randomized Gelis trial. J Clin Med. 2019;8(6):876-93.

31. Curran GM, Bauer M, Mittman B, Pyne JM, Stetler C. Effectivenessimplementation hybrid designs: combining elements of clinical effectiveness and implementation research to enhance public health impact. Med Care. 2012;50(3):217-26.

32. Palinkas LA, Mendon SJ, Hamilton AB. Innovations in mixed methods evaluations. Annu Rev Public Health. 2019;40:423-42.

33. Innovationsausschuss beim Gemeinsamen Bundesausschuss. Mind: Pregnancy - Online-basierte Achtsamkeitsintervention zur Stärkung psychischer Stabilität von Schwangeren und Förderung einer physiologischen Geburt 2019 [30.10.2019]. Available from: https:// innovationsfonds.g-ba.de/projekte/neue-versorgungsformen/mindpregnancy-online-basierte-achtsamkeitsintervention-zur-staerkungpsychischer-stabilitaet-von-schwangeren-und-foerderung-einerphysiologischen-geburt.179.

34. Flothkötter M, Kauth T, Koletzko B, Korsten-Reck U, Kottenkamp W, Lambeck A, et al. Dokumentation zum Projekt "9+12 Gemeinsam gesund in Schwangerschaft und erstem Lebensjahr". Berlin: Plattform Ernährung und Bewegung e.V; 2014

35. Koletzko B, Bauer C-P, Cierpka M, Cremer M, Flothkötter M, Graf C, et al. Ernährung und Bewegung von Säuglingen und stillenden Frauen. Monatsschrift Kinderheilkunde. 2016;164(9):771-98.

36. Koletzko B, Cremer M, Flothkotter M, Graf C, Hauner H, Hellmers C, et al. Diet and lifestyle before and during pregnancy - practical recommendations of the Germany-wide healthy start - young family network. Geburtshilfe Frauenheilkd. 2018;78(12):1262-82.

37. Hettema JE, Hendricks PS. Motivational interviewing for smoking cessation: a meta-analytic review. J Consult Clin Psychol. 2010;78(6):868-84.

38. Poirier MK, Clark MM, Cerhan JH, Pruthi S, Geda YE, Dale LC. Teaching motivational interviewing to first-year medical students to improve counseling skills in health behavior change. Mayo Clin Proc. 2004;79(3):327-31.

39. Miller WR, Rollnick S. Motivational interviewing: helping people change. 3rd ed. New York: Guilford Press; 2013.

40. Bollmeier N, Bonfig J, Cremer M, Ferrari N, Floto-Stammen S. Ernährung und Bewegung in der Schwangerschaft: Referentenhandbuch zur Multiplikatorenfortbildung für Fachkräfte. Bonn: Aid Infodienst Ernährung Landwirtschaft Verbraucherschutz e.V; 2014

41. Miller WR, Rollnick S. Motivierende Gesprächsführung. Freiburg: Lambertus Verlag $\mathrm{GmbH} ; 2015$.

42. Rosengren D. Arbeitsbuch Motivierende Gesprächsführung. Lichtenau/ Westfalen Probst-Verlag; 2015.

43. Demmel R, Kemény G. Motivational interviewing box mit Fragekarten. Freiburg im Breisgau: Freiburg; 2017.

44. Glasgow RE, Vogt TM, Boles SM. Evaluating the public health impact of health promotion interventions: the RE-AIM framework. Am J Public Health. 1999;89(9):1322-7.

45. Gaglio B, Shoup JA, Glasgow RE. The RE-AIM framework: a systematic review of use over time. Am J Public Health. 2013;103(6):38-46.

46. Institute of Medicine (US) and National Research Council (US) Committee to Reexamine IOM Pregnancy Weight Guidelines. Weight Gain During Pregnancy: Reexamining the Guidelines. 2009. Available from: https://www. ncbi.nlm.nih.gov/books/NBK32813/. 
47. Chasan-Taber L, Schmidt MD, Roberts DE, Hosmer D, Markenson G, Freedson PS. Development and validation of a pregnancy physical activity questionnaire. Med Sci Sports Exerc. 2004;36(10):1750-60.

48. Studie zur Gesundheit von Kindern und Jugendlichen. Elternfragebogen 0-2. Berlin: Robert-Koch Institut; 2003.

49. Haftenberger M, Heuer T, Heidemann C, Kube F, Krems C, Mensink GB. Relative validation of a food frequency questionnaire for national health and nutrition monitoring. Nutr J. 2010;9:36.

50. Kromeyer-Hauschild K, Wabitsch M, Kunze D, Geller F, Geiß HC, Hesse V, et al. Perzentile für den Body-mass-Index für das Kindes- und Jugendalter unter Heranziehung verschiedener deutscher Stichproben. Monatsschrift Kinderheilkunde. 2001;149(8):807-18.

51. Reilly JJ, Kelly J, Wilson DC. Accuracy of simple clinical and epidemiological definitions of childhood obesity: systematic review and evidence appraisal. Obes Rev. 2010;11(9):645-55.

52. WHO Multicentre Growth Reference Study Group. WHO Child Growth Standards based on length/height, weight and age. Acta Paediatr Suppl. 2006;450:76-85.

53. Mensink GB, Burger M. Was isst du? Ein Verzehrshäufigkeitsfragebogen für Kinder und Jugendliche. Bundesgesundheitsblatt-GesundheitsforschungGesundheitsschutz. 2004;47(3):219-26.

54. Musher-Eizenman D, Holub S. Comprehensive feeding practices questionnaire: validation of a new measure of parental feeding practices. $J$ Pediatr Psychol. 2007;32(8):960-72.

55. Flottorp SA, Oxman AD, Krause J, Musila NR, Wensing M, Godycki-Cwirko M, et al. A checklist for identifying determinants of practice: a systematic review and synthesis of frameworks and taxonomies of factors that prevent or enable improvements in healthcare professional practice. Implement Sci. 2013;8:35

56. Drummond MF, Sculpher MJ, Torrance GW, O'Brien BJ, Stoddart GL. Methods for the Economic Evaluation of Health Care Programmes. New York: Oxford University Press; 2005.

57. Trueman P, Anokye NK. Applying economic evaluation to public health interventions: the case of interventions to promote physical activity. J Public Health (Oxf). 2013;35(1):32-9.

58. Alayli-Goebbels AFG, Dellaert BGC, Knox SA, Ament AJHA, Lakerveld J, Bot $\mathrm{SDM}$, et al. Consumer preferences for health and nonhealth outcomes of health promotion: results from a discrete choice experiment. Value Health. 2013;16(1):114-23.

59. Rauh K, Gabriel E, Kerschbaum E, Schuster T, von Kries R, Amann-Gassner U, et al. Safety and efficacy of a lifestyle intervention for pregnant women to prevent excessive maternal weight gain: a cluster-randomized controlled trial. BMC Pregnancy Childbirth. 2013;13:151.

60. Gale NK, Heath G, Cameron E, Rashid S, Redwood S. Using the framework method for the analysis of qualitative data in multi-disciplinary health research. BMC Med Res Methodol. 2013;13:117.

61. Hill B, McPhie S, Moran $\sqcup$, Harrison P, Huang TT, Teede H, et al. Lifestyle intervention to prevent obesity during pregnancy: implications and recommendations for research and implementation. Midwifery. 2017:49:13-8

62. AQUA - Institut für angewandte Qualitätsförderung und Forschung im Gesundheitswesen GmbH. Bundesauswertung zum Erfassungsjahr 2014. Göttingen: 16/1 - Geburtshilfe Qualitätsindikatoren; 2015.

63. Rattay P, Starker A, Domanska O, Butschalowsky H, Gutsche J, Kamtsiuris $P$. Trends in der Inanspruchnahme ambulant-ärztlicher Leistungen im Kindes- und Jugendalter Ergebnisse der KiGGS-Studie - Ein Vergleich von Basiserhebung und erster Folgebefragung (KiGGS Welle 1). Bundesgesundheitsbl Gesundheitsforsch Gesundheitsschutz. 2014;57: 878-91.

64. Yang N, Ginsburg GS, Simmons LA. Personalized medicine in women's obesity prevention and treatment: implications for research, policy and practice. Obes Rev. 2013;14(2):145-61.

65. Heckman CJ, Egleston BL, Hofmann MT. Efficacy of motivational interviewing for smoking cessation: a systematic review and meta-analysis. Tob Control. 2010;19(5):410-6.

66. Armstrong MJ, Mottershead TA, Ronksley PE, Sigal RJ, Campbell TS, Hemmelgarn BR. Motivational interviewing to improve weight loss in overweight and/or obese patients: a systematic review and meta-analysis of randomized controlled trials. Obes Rev. 2011;12(9):709-23.

67. Samdal GB, Eide GE, Barth T, Williams G, Meland E. Effective behaviour change techniques for physical activity and healthy eating in overweight and obese adults; systematic review and meta-regression analyses. Int J Behav Nutr Phys Act. 2017;14(1):42.
68. Van der Pligt P, Willcox J, Hesketh KD, Ball K, Wilkinson S, Crawford D, et al. Systematic review of lifestyle interventions to limit postpartum weight retention: implications for future opportunities to prevent maternal overweight and obesity following childbirth. Obes Rev. 2013;14(10):792-805.

69. Heslehurst N, Crowe L, Robalino S, Sniehotta FF, McColl E, Rankin J. Interventions to change maternity healthcare professionals' behaviours to promote weight-related support for obese pregnant women: a systematic review. Implement Sci. 2014;9(1):97.

70. Olander EK, Atkinson L, Edmunds JK, French DP. The views of pre- and postnatal women and health professionals regarding gestational weight gain: an exploratory study. Sex Reprod Healthc. 2011;2(1):43-8.

71. Schmied VA, Duff M, Dahlen HG, Mills AE, Kolt GS. 'Not waving but drowning': a study of the experiences and concerns of midwives and other health professionals caring for obese childbearing women. Midwifery. 2011; 27(4):424-30.

72. Okorodudu DE, Bosworth HB, Corsino L. Innovative interventions to promote behavioral change in overweight or obese individuals: a review of the literature. Ann Med. 2015;47(3):179-85.

73. O'Brien OA, McCarthy M, Gibney ER, McAuliffe FM. Technology-supported dietary and lifestyle interventions in healthy pregnant women: a systematic review. Eur J Clin Nutr. 2014;68(7):760-6.

74. Richardson CR, Newton TL, Abraham JJ, Sen A, Jimbo M, Swartz AM. A meta-analysis of pedometer-based walking interventions and weight loss. Ann Fam Med. 2008;6(1):69-77.

75. Wu T, Gao X, Chen M, van Dam RM. Long-term effectiveness of diet-plusexercise interventions vs. diet-only interventions for weight loss: a metaanalysis. Obes Rev. 2009;10(3):313-23.

\section{Publisher's Note}

Springer Nature remains neutral with regard to jurisdictional claims in published maps and institutional affiliations.
Ready to submit your research? Choose BMC and benefit from:

- fast, convenient online submission

- thorough peer review by experienced researchers in your field

- rapid publication on acceptance

- support for research data, including large and complex data types

- gold Open Access which fosters wider collaboration and increased citations

- maximum visibility for your research: over $100 \mathrm{M}$ website views per year

At $\mathrm{BMC}$, research is always in progress.

Learn more biomedcentral.com/submissions 